\title{
Immunoproteomics of Plasmodium falciparum-infected red blood cell membrane fractions
}

\author{
Fernanda J Cabral ${ }^{1,2 /+}$, Luciana G Vianna ${ }^{3}$, Marcia M Medeiros ${ }^{2,6}$, \\ Bianca Cechetto Carlos ${ }^{2}$, Rosimeire D Martha ${ }^{4}$, Nadia Maria Silva', \\ Luiz Hildebrando P da Silva ${ }^{4,+}$, Rodrigo G Stabeli ${ }^{5}$, Gerhard Wunderlich ${ }^{2}$
}

\author{
${ }^{1}$ Universidade Estadual de Campinas, Instituto de Biologia, Departamento de Biologia Animal, Campinas, SP, Brasil \\ ${ }^{2}$ Universidade de São Paulo, Instituto de Ciências Biomédicas, Departamento de Parasitologia, São Paulo, SP, Brasil \\ ${ }^{3}$ Instituto Butantan, São Paulo, SP, Brasil \\ ${ }^{4}$ Centro de Pesquisa em Medicina Tropical, Porto Velho, RO, Brasil \\ ${ }^{5}$ Fundação Oswaldo Cruz-Fiocruz, Rio de Janeiro, RJ, Brasil \\ ${ }^{6}$ Universidade Nova de Lisboa, Instituto de Higiene e Medicina Tropical, Lisboa, Portugal
}

BACKGROUND The surface of infected red blood cells (iRBCs) has been widely investigated because of the molecular complexity and pathogenesis mechanisms involved. Asymptomatic individuals are important in the field because they can perpetuate transmission as natural reservoirs and present a challenge for diagnosing malaria because of their low levels of circulating parasites. Recent studies of iRBC antibody recognition have shown that responses are quantitatively similar in symptomatic and asymptomatic infections, but no studies have characterised the plasmodial proteins targeted by this response.

OBJECTIVES Our main objective was to identify Plasmodium falciparum proteins associated with iRBC ghosts recognised by antibodies in the sera of symptomatic and asymptomatic individuals in the Brazilian Amazon.

METHODS We collected symptomatic and asymptomatic sera from patients residing in the Brazilian Amazon and P. falciparum iRBC ghosts to identify the proteins involved in natural antibody recognition by 2D-electrophoresis, western blotting, and highresolution mass spectrometry.

FINDINGS 2D gel-based immunoproteome analysis using symptomatic and asymptomatic sera identified 11 proteins with at least one unique peptide, such as chaperones HSP70-1 and HSP70-x, which likely are components of the secretion machinery/PTEX translocon. PfEMP1 is involved in antigenic variation in symptomatic infections and we found putative membrane proteins whose functions are unknown.

MAIN FINDINGS Our results suggest a potential role of old and new proteins, such as antigenic variation proteins, iRBC remodelling, and membrane proteins, with no assigned functions related to the immune response against $P$. falciparum, providing insights into the pathogenesis, erythrocyte remodelling, and secretion machinery important for alternative diagnosis and/or malaria therapy.

Key words: Plasmodium falciparum - symptomatic - asymptomatic - proteomics

Plasmodium falciparum is the causative agent of malaria in tropical areas of the world. The World Health Organization (WHO) recently estimated that approximately 214 million people were infected by Plasmodium and 438,000 people died from malaria in 2015 (WHO 2015). Asymptomatic malaria is often characterised by the submicroscopic presence of parasites in the blood of persons with no presence of symptoms. Epidemiological data have revealed the importance of asymptomatic infections in malaria transmission (Alves et al. 2005) and high prevalence of asymptomatic infections in the Brazilian Amazon (Alves et al. 2002, da Silva-Nunes et al. 2012). Although the importance of asymptomatic epidemiology has been well-demonstrated

doi: 10.1590/0074-02760170041

Financial support: CAPES, CNPq, FAPESP

FJC was a Pos-Doc NanoCAPES fellow, GW is a CNPq research fellow and supported by FAPESP (grant 2012/23306-5)

+ Corresponding author: fjanku@unicamp.br

+ In memoriam

Received 1 February 2017

Accepted 30 June 2017 in the Brazilian Amazon, few studies have examined the pathogenesis and antigen recognition in asymptomatic infections. Recently, two studies demonstrated the important aspects of patient serum antibodies in antigen recognition from asymptomatic infections in the Brazilian Amazon. Recombinant expression of merozoite antigens and ultimately recognition of recombinant antigens by symptomatic and asymptomatic plasma antibodies showed that merozoite antigen recognition occurred regardless of symptoms and that other factors may contribute to clinical protection acquisition (Medeiros et al. 2013). Another study correlating the symptomatic/ asymptomatic status with infected red blood cell (iRBC) recognition found no striking difference in the frequency and intensity of antibody recognition (Fratus et al. 2014). In contrast, several studies showed that plasmodial proteins displayed on iRBCs are responsible for: (i) targeted antigen recognition and associated immunity and (ii) immune system escape by antigenic variation (Leech et al. 1984, Cheng et al. 1998, Winter et al. 2005, Chan et al. 2012). The well-known variant surface antigens (VSAs), which are related to pathogenesis and antigenic variation, are $P$. falciparum erythrocyte 
membrane protein 1 (PfEMP1) (Bull et al. 1998), Rifin (repetitive interspersed family) (Kyes et al. 1999), and possibly surface-associated interspersed genes (surfins) (Winter et al. 2005). A recent study showed that PfEMP1 is the main target of naturally acquired antibodies and is associated with protection from age-related clinical manifestations in symptomatic infections (Chan et al. 2012). However, no studies have examined the differences in $\mathrm{iRBC}$ recognition by antibodies in (oligo)symptomatic and asymptomatic infections. Additionally, it is unknown which proteins/peptides are the major targets in the recognition by natural antibodies in symptomatic/ asymptomatic infections in the Brazilian Amazon. In this study, we applied mass spectrometry analysis using iRBC ghosts, which have infected red blood cell plasma membranes, the erythrocyte's submembrane skeleton, Maurer's clefts, and the protein transport machinery of the parasite to determine which molecules are recognised by symptomatic/asymptomatic serum.

To address the immunproteome, $P$. falciparum patient field isolate 112 (2006) was collected from Rondonia state, Brazil. Parasites were cultured in a candle jar (Jensen \& Trager 1977) in RPMI 1640 medium containing 10\% human plasma B and B+ erythrocytes. All procedures involving human participants were conducted in accordance with the ethical standards of the Institute of Biomedical Sciences of University of São Paulo research committee (protocol CEPSH 041.11). For assays, parasites were floated in $6 \%$ Voluven (Fresenius Kabi, Campinas, Brazil, Lelièvre et al. 2005). After floating for 24-36 h, trophozoites were collected and the erythrocytes were lysed with $0.2 x$ hypotonic solution as described previously (Rabilloud et al. 1999) and iRBC ghosts were stored at $-80^{\circ} \mathrm{C}$ until use. 2D-electrophoresis was conducted in triplicate, and each experiment involved the use of two gels where one was stained with Coomassie blue and the other was transferred onto a membrane and incubated with antibodies. Lysed red blood cell membranes were used as negative controls, and western blotting with symptomatic patient sera (pool of $n=20$ ) and asymptomatic carrier sera (pool of $n=20)$ revealed no bands (data not shown). Isoelectric focusing was performed as described. Briefly, proteins were homogenised in De-Streak (GE Healthcare, Little Chalfont, UK) buffer and incubated with a $7-\mathrm{cm}$ immobilised $\mathrm{pH}$ gradient strip overnight. The following day, the strips were subjected to isoelectric focusing in an ETTAN IPGPHOR (GE Healthcare) apparatus, with a total accumulation of $15,702 \mathrm{Vh}$ for $7 \mathrm{~h}$. For the second dimension, strips were first reduced by 15 min under mild agitation in equilibration buffer $(2 \% \mathrm{SDS}, 6 \mathrm{M}$ urea, $75 \mathrm{mM}$ Tris- $\mathrm{HCl}(\mathrm{pH} 8,8)$ containing $30 \%$ glycerol and $0.002 \%$ bromophenol blue) and $1 \%$ DTT. After reduction, the strips were placed in $0.25 \%$ iodoacetamide in the same buffer for $15 \mathrm{~min}$. Electrophoreses were performed in ZOOM 4-20\% Tris-glicine gels and in ZOOM 4-20\% Bistris gels (Invitrogen, Carlsbad, CA, USA) and stained with Coomassie blue. The resulting gels were maintained in $20 \%$ ammonium sulphate until spots were picked. Patient sera were obtained as per the methods described by Medeiros et al. (2013) and Carlos et al. (2016). Western blotting was performed using 1:1000 dilutions of pooled patient sera. The protein spots were recognised, picked from the (NH4) ${ }_{2} \mathrm{SO}_{4}$-soaked replica gel, reduced, alkylated, and digested overnight with $12.5 \mathrm{ng} / \mu \mathrm{L}$ of trypsin (sequencing grade, Promega, Madison, WI, USA) (Shevchenko et al. 1996) according to the manufacturer's instructions. Extracted peptides were desalted in reversed phase ziptips before liquid chromatography-mass spectrometry (LC$\mathrm{MS} / \mathrm{MS}$ ) analysis. MS analyses were performed on an LTQ-Orbitrap Velos ETD (Thermo Fisher Scientific): (Thermo Scientific 2012) coupled with an Easy nanoLC II (Thermo Fisher Scientific). The peptides were separated on a C18RP column over a 45-min gradient. Database searching was performed using the SEQUEST algorithm in Proteome discoverer version 1.4 (Thermo Fisher Scientific) against PlasmoDB data (plasmoDB.org) for P. falciparum 3D7 and P. falciparum IT. The instrument conditions were checked using 50 fmol of a tryptic digest of bovine serum albumin as a standard. Sample carryover was completely removed between runs. All samples were evaluated in duplicate. Sequence analyses of the molecular function, cellular component, and biological processes were conducted using the Gene Ontology (GO) tool on Gene DB databank (www.genedb.org).

We identified five spots in symptomatic sera and six spots in asymptomatic sera (Tables I-II). In Figure (A), a typical result of the recognition profile of the symptomatic sera pool is shown. The identification results are shown in Table I. Analysis of the confidence of the peptides were evaluated based on decoy rates, which represent sequenced peptides with false discovery rates $<1 \%$ and confident protein identification with at least a minimum of one unique peptide and one peptide sequence matching (PSM) according to the manufacturer instructions (Thermo Fisher Scientific) (Tables I-II). We identified PfEMP1 (PF3D7_0533100) annotated as
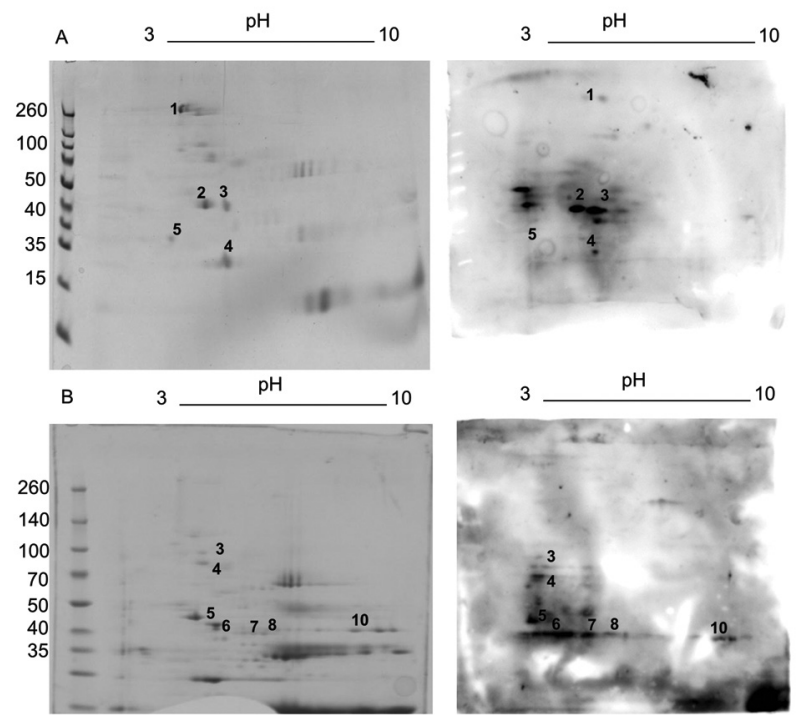

Ghost extracts analysed by 2D-electrophoresis. (A) Right: replica gel of Plasmodium falciparum proteins stained with Coomassie blue; left: western blotting with the sera of symptomatic patients. (B) Right: replica gel of $P$. falciparum proteins, stained with Coomassie blue; left: western blotting with the sera of asymptomatic patients from an endemic area. 


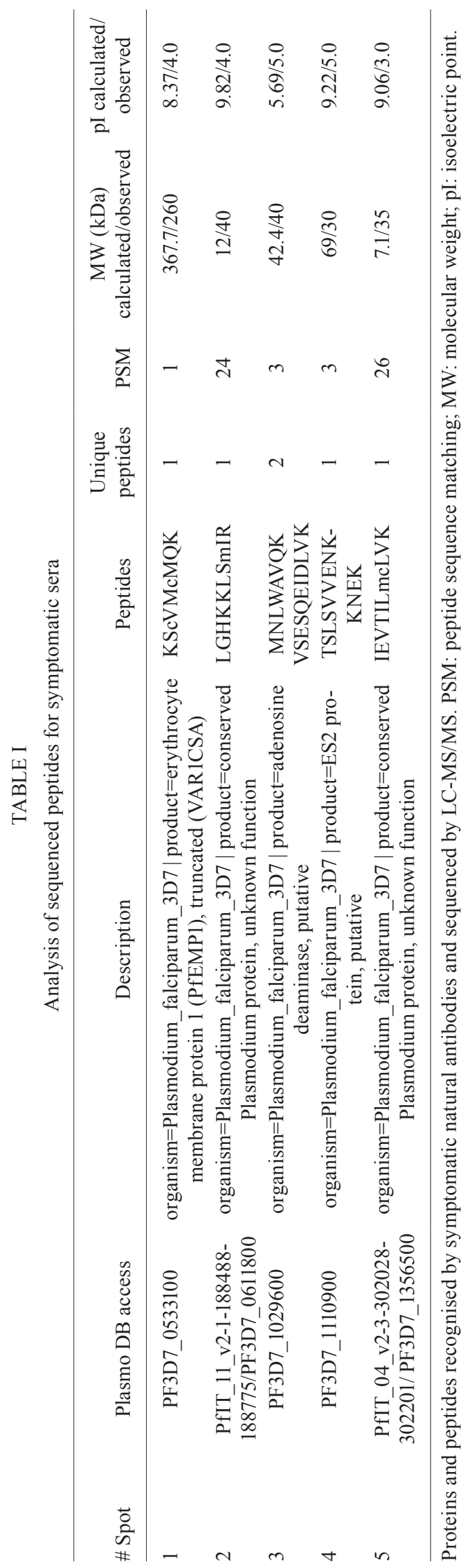

truncated varlcsa which had one unique peptide and one PSM, reflecting the low abundance of this protein in the sample (Table I). Other proteins were sequenced, such as adenosine deaminase (PF3D7 1029600) with two unique peptides. However, this protein may be a contamination of our ghost preparation with the cellular fraction of the parasite, as this protein has neither an export motif nor any associated function with the iRBC ghost (Table I). Sequencing of another protein (PF3D7 1356500) with 26 PSMs and Gene Ontology (GO) analysis indicated that this protein plays a role in the Golgi network membrane and/or is an integral membrane protein (Table III). Because these peptides were apparently recognised by naturally acquired antibodies, they may be membrane proteins involved in immunity, infection, and/or clinical manifestations.

The results obtained from the recognition of proteins by sera from asymptomatic persons revealed no striking differences compared with the symptomatic sera recognition profile (Table II). Sera from asymptomatic individuals showed seven spots but it was identified by MS six spots [Figure (B)]. Similarly, some proteins were annotated as proteins with unknown function (PF3D7_0918400 and PF3D7_1369400) and GO analysis showed that these are putative membrane proteins (Table IV). Two other proteins, PF3D7_1023600 and PF3D7_1007800, were identified and are annotated as proteins with unknown function. GO analysis revealed that these proteins are cytoplasmic proteins (Table IV).

We found several hits for heat shock proteins, such as HSP70-1 (PF3D7 0818900) and HSP70-X (PF3D7 0831700), and both were recognised by antibodies from asymptomatic carriers (Tables II-IV). 2D-electrophoresis showed that PfHSP70 and PfHSP70-X have a molecular weight of approximately $75 \mathrm{kDa}$ and $\mathrm{pI}$ of 5.5, and were strongly recognised in western blotting (Figure). These data agree with a previous study showing that specific antisera against a PfHSP70-x strongly recognise a 2D-electrophoresis spot of $72.3 \mathrm{kDa}$ with a $\mathrm{pI}$ of 5.4 (Grover et al. 2013). Further, GO analysis showed that PfHSP70-x is related to the PTEX complex and is a component of the iRBC cell cytosol (Table IV).

$P$. falciparum iRBC membrane ghosts were previously defined as follow: an iRBC membrane plus Maurer's clefts components, secreted proteins of the parasite to secreted into the cytosol of erythrocytes and erythrocyte submembrane skeleton (Blisnick et al. 2000). We detected proteins related to the membrane, cytoplasm, and erythrocyte cytosol. The predicted secretome of $P$. falciparum indicates that the parasite secretes more than 320 proteins into the erythrocyte cytosol which plays a role in antigenic variation and host erythrocyte remodelling (Hiller et al. 2004, Marti et al. 2004, Maier et al. 2009).

To enable erythrocyte remodelling and presentation of its own antigens on the erythrocyte membrane surface, $P$. falciparum translocates proteins from the parasitophorous vacuole to the erythrocyte membrane via PTEX (Elsworth et al. 2014) translocon, and PfHSP40 and PfHSP70 may be involved in protein assembly after translocation. Functionally, molecular chaperones are important in the remodelling and folding of proteins that are transported into iRBCs (de Koning-Ward et al. 2009, Grover et al. 2013). In this study, we found that PfHSP70 and PfHSP70x 


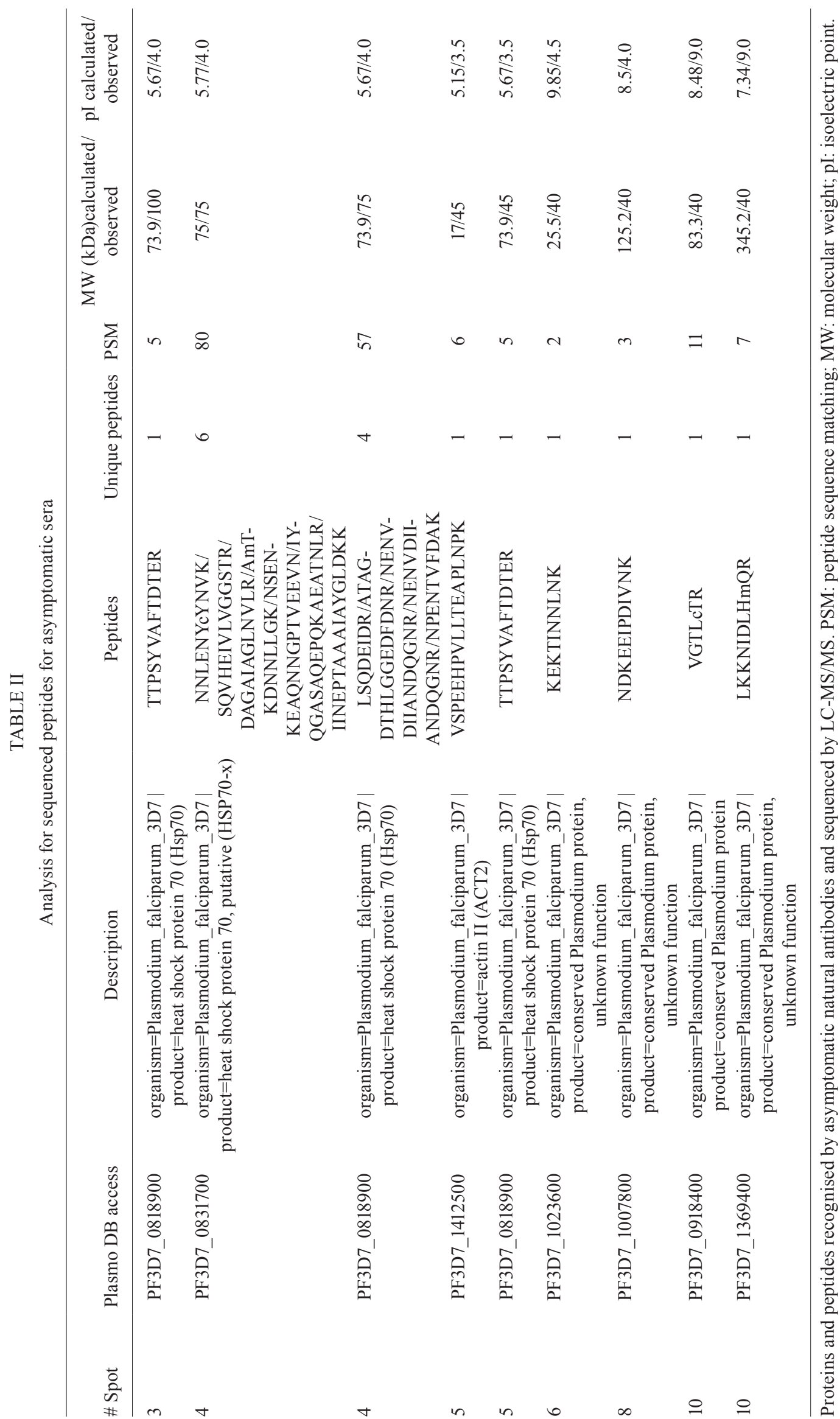



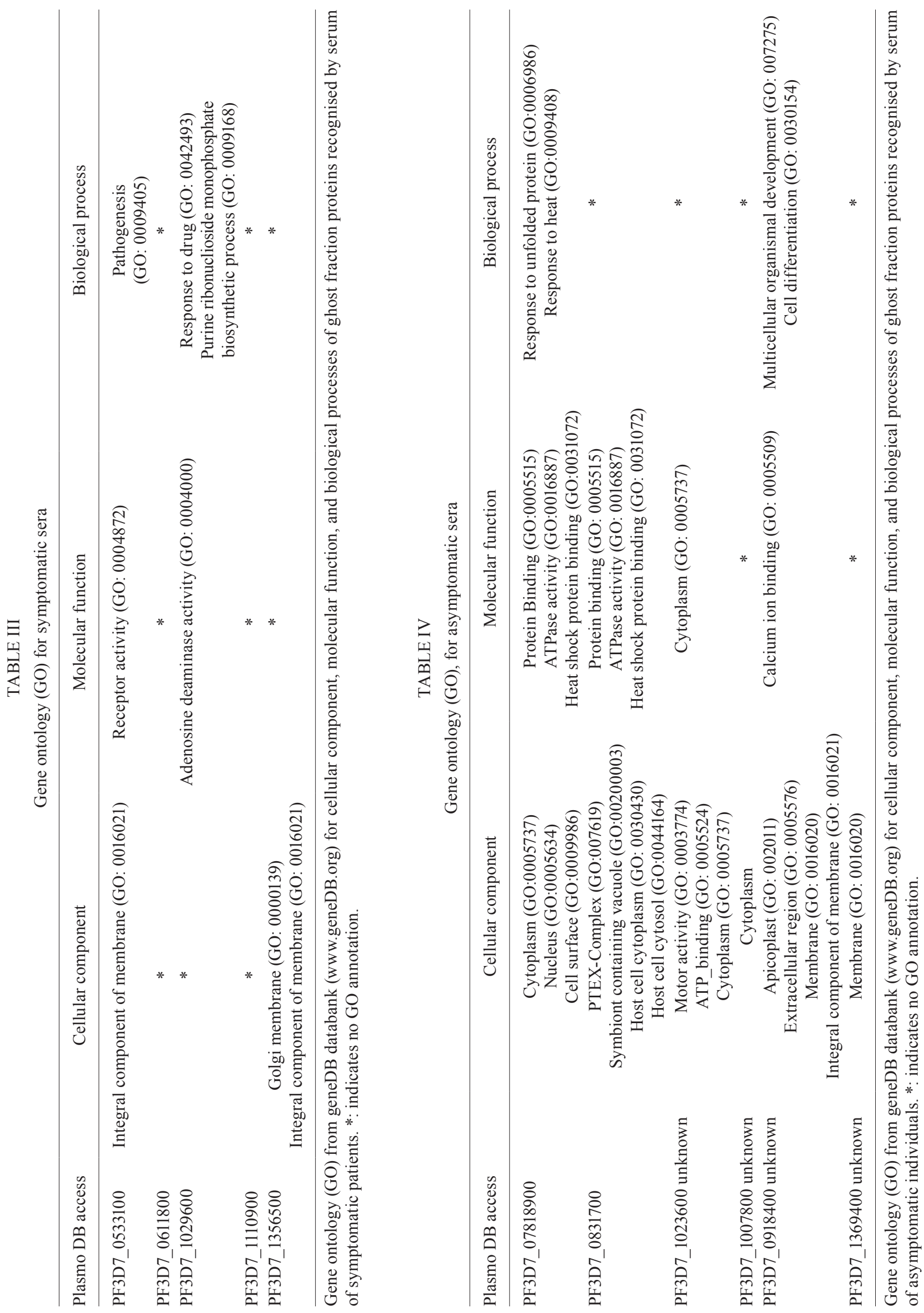
are highly recognised by antibodies in asymptomatic sera. In P. falciparum, PfHSP70-1 was identified using a proteomic approach as part of Maurer's clefts (Vincensini et al. 2005), although this protein is also highly abundant in the cytosol and parasitophorous vacuole (Pesce \& Blatch 2014). Because of its high abundance and importance in protein translocation and serum recognition in our study and others (Vincensini et al. 2005), this protein is a candidate for new drug interventions for malaria treatment to control the parasite in the circulation. Our main finding regarding chaperones is that the HSP70-x sequence contained six unique peptides. Recent studies showed that PfHSP70-x is located in the erythrocyte cytosol and may play a role in trafficking of PfEMP1 to the iRBC surface by forming chaperone complexes/co-complexes within the host erythrocyte (Kulzer et al. 2012). Trafficking of HSP70 $\mathrm{x}$ is dependent on the PTEX translocon (Rhiel et al. 2016). Our findings for PfHSP70-x suggest that asymptomatic individuals mount an antibody response against chaperone complexes, but the importance of this response remains unclear.

In this study, GO analysis revealed that some proteins are putatively localised on the erythrocyte membrane. Sera from symptomatic patients recognised the protein product of PF3D7_0533100, annotated as PfEMP1 (varlcsa). PfEMP1 is well-known as part of the iRBC membrane and is highly associated with malaria pathogenesis and cytoadherence; thus, it is not surprising that symptomatic serum recognised this protein. varlcsa expression, based on transcript levels, does not appear to be involved in the allelic exclusion of var transcription (Kyes et al. 2003). Additionally, varlcsa is annotated as a pseudogene in the 3D7 strain, IT strain, and P. reichenowi, and thus the importance of these results is unclear. Here, the field isolate 112 was used as a source of protein, and it is possible that either varlcsa is functional in this strain or that other PfEMP1 proteins in this strain contain the peptide sequence. Other prominent and surface exposed antigens such as surfins, rifins, and other PfEMP1s were poorly identified or not identified at all in this study because they were present at very low concentration in our preparations of iRBC ghosts. In addition, when analysing proteins recognised by sera from asymptomatic patient gels, PfEMP1 identification may have failed on account of using bis-Tris gels to resolve proteins by electrophoresis. Another study showed that PfEMP-1 detection by western blotting shows better results when performed using pre-cast Tris-glycine gels or Tris acetate gels (Chan et al. 2012). Even though we successfully identify main virulence antigens, the influence of these antigens on the immune response should not be discarded in both symptomatic and asymptomatic infections.

The humoral immune response against target proteins also depends on the genetic host background and the ability to switch between antibody subclasses. A recent study correlating protein microarray results to immune reactions to $\operatorname{IgG}$ and $\operatorname{IgM}$ in Africa revealed that several proteins in the parasite are targets of both $\mathrm{IgG} / \mathrm{IgM}$ responses or uniquely to $\mathrm{IgG}$ or IgM. These authors also found that host genetic resistance to malaria is related to the ability to produce immune reactions against several proteins of the parasite (Arama et al. 2015). When we compared proteins recognised by asymptomatic carrier antibodies to those recognised by $\operatorname{IgG}$ and $\operatorname{IgM}$ in the protein array (Arama et al. 2015), we found that similar proteins (PF3D7_0818900, PF3D7_0831700) were recognised in both the previous study and our study. Another study conducted in the western Amazon using the same sera as used in this study revealed the prevalence of the $\operatorname{IgG}$ 1 subclass against surfin proteins in asymptomatic cases, and this tendency was maintained independently of the age of the individual from whom the serum was collected (M Medeiros, unpublished observations). These results indicate that for some individuals, natural resistance to infection and the presence of symptoms arise in early childhood; however, additional studies regarding $\operatorname{IgG}$ subclasses and IgM in relation to the protection from symptoms of malaria should be conducted in the Brazilian Amazon to confirm this hypothesis.

The main finding of this study is that proteins related to iRBC ghosts may be important factors in antibody responses and are correlated to the presence and absence of malaria symptoms. The identified antigens may influence the outcome of malaria and are potential targets of future studies on their function and localisation.

\section{ACKNOWLEDGEMENTS}

To Dr Norma Yamanouye for assistance with 2D-eletrophoresis analyses. The authors thank Dr William Festuccia and Dr Giuseppe Palmisano for providing reagents and analysis support, respectively; Wolfgang Fischer for technical assistance; CEFAPUSP and CNPEM-LNBio for mass spectrometry analysis.

\section{AUTHORS' CONTRIBUTION}

GW, RGS and LHPS conceived the study design; FJC, LGV, MMM, BCC and RDM performed the experiments; FJC and NMS analysed the results; FJC and GW wrote and revised the paper.

\section{REFERENCES}

Alves FP, Durlacher RR, Menezes MJ, Krieger H, Silva LH, Camargo EP. High prevalence of asymptomatic Plasmodium vivax and Plasmodium falciparum infections in native Amazonian populations. Am J Trop Med Hyg. 2002; 66(6): 641-8.

Alves FP, Gil LH, Marrelli MT, Ribolla PE, Camargo EP, Silva LH. Asymptomatic carriers of Plasmodium spp. as infection source for malaria vector mosquitoes in the Brazilian Amazon. J Med Entomol. 2005; 42(5): 777-9.

Arama C, Skinner J, Doumtabe D, Portugal S, Tran TM, Jain A, et al. Genetic resistance to malaria is associated with greater enhancement of immunoglobulin (Ig)M than IgG responses to a broad array of Plasmodium falciparum antigens. Open Forum Infect Dis. 2015; 2(3): ofv118.

Blisnick T, Betoulle MEM, Barale JC, Uzureau P, Berry L, Desroses $\mathrm{S}$, et al. Pfsbp1, a Maurer's cleft Plasmodium falciparum protein, is associated with the erythrocyte skeleton. Mol Biochem Parasitol. $2000 ; 111(1): 107-21$.

Bull PC, Lowe BS, Kortok M, Molyneux CS, Newbold CI, Marsh K. Parasite antigens on the infected red cell surface are targets for naturally acquired immunity to malaria. Nat Med. 1998; 4(3): 358-60.

Carlos BC, Fotoran WL, Menezes MJ, Cabral FJ, Bastos MF, Costa FT, et al. Expressed var gene repertoire and variant surface antigen diversity in a shrinking Plasmodium falciparum population. Exp Parasitol. 2016; 170: 90-9. 
Chan JA, Howell KB, Reiling L, Ataide R, Mackintosh CL, Fowkes FJ, et al. Targets of antibodies against Plasmodium falciparuminfected erythrocytes in malaria immunity. J Clin Invest. 2012; 122(9): 3227-38.

Cheng Q, Cloonan N, Fischer K, Thompson J, Waine G, Lanzer M, et al. Stevor and Rif are Plasmodium falciparum multicopy gene families which potentially encode variant antigens. Mol Biochem Parasitol. 1998; 97: 161-76.

da Silva-Nunes M, Moreno M, Conn JE, Gamboa D, Abeles S, Vinetz $\mathrm{JM}$, et al. Amazonian malaria: asymptomatic human reservoirs, diagnostic challenges, environmentally driven changes in mosquito vector populations, and the mandate for sustainable control strategies. Acta Trop. 2012; 121(3): 281-91.

de Koning-Ward TF, Gilson PR, Boddey JA, Rug M, Smith BJ, Papenfuss AT, et al. A newly discovered protein export machine in malaria parasites. Nature. 2009; 459: 945-9.

Elsworth B, Matthews K, Nie CQ, Kalanon M, Charnaud SC, Sanders $\mathrm{PR}$, et al. PTEX is an essential nexus for protein export in malaria parasites. Nature. 2014; 511(7511): 587-91.

Fratus ASB, Cabral FJ, Fotoran WL, Medeiros MM, Carlos BC, Martha $\mathrm{RD}$, et al. Antibody recognition of Plasmodium falciparum infected red blood cells by symptomatic and asymptomatic individuals in the Brazilian Amazon. Mem Inst Oswaldo Cruz. 2014; 109(5): 598-607.

Grover M, Chaubey S, Ranade S, Tatu U. Identification of an exported heat shock protein 70 in Plasmodium falciparum. Parasite. 2013; 20: 2.

Hiller NL, Bhattacharjee S, van Ooij C, Liolios K, Harrison T, LopezEstrano C, et al. A host-targeting signal in virulence proteins reveals a secretome in malarial infection. Science. 2004; 306(5703): 1934-7.

Jensen JB, Trager W. Plasmodium falciparum in culture: use of outdated erthrocytes and description of the candle jar method. J Parasitol. 1977; 63(5): 883-6.

Kulzer S, Charnaud S, Dagan T, Riedel J, Mandal P, Pesce ER, et al. Plasmodium falciparum-encoded exported hsp70/hsp40 chaperone/co-chaperone complexes within the host erythrocyte. Cell Microbiol. 2012; 14(11): 1784-95.

Kyes SA, Christodoulou Z, Raza A, Horrocks P, Pinches R, Rowe JA, et al. A well-conserved Plasmodium falciparum var gene shows an unusual stage-specific transcript pattern. Mol Microbiol. 2003; 48(5): 1339-48.

Kyes SA, Rowe JA, Kriek N, Newbold CI. Rifins: a second family of clonally variant proteins expressed on the surface of red cells infected with Plasmodium falciparum. Proc Natl Acad Sci USA. 1999; 96(16): 9333-8.
Leech JH, Barnwell JW, Miller LH, Howard RJ. Identification of a strainspecific malarial antigen exposed on the surface of Plasmodium falciparum-infected erythrocytes. J Exp Med. 1984; 159(6): 1567-75.

Lelièvre J, Berry A, Benoit-Vical F. An alternative method for Plasmodium culture synchronization. Exp Parasitol. 2005; 109(3): 195-7.

Maier AG, Cooke BM, Cowman AF, Tilley L. Malaria parasite proteins that remodel the host erythrocyte. Nat Rev Microbiol. 2009; 7(5): 341-54.

Marti M R, Good T, Rug M, Knuepfer E, Cowman AF. Targeting malaria virulence and remodeling proteins to the host erythrocyte. Science. 2004; 306(5703): 1930-3.

Medeiros MM, Fotoran WL, dalla Martha RC, Katsuragawa TH, da Silva LHP, Wunderlich G. Natural antibody response to Plasmodium falciparum merozoite antigens MSP5, MSP9 and EBA175 is associated to clinical protection in the Brazilian Amazon. BMC Infect Dis. 2013; 13: 608.

Pesce ERB, Blatch GL. Plasmodial Hsp40 and Hsp70 chaperones: current and future perspectives. Parasitology. 2014; 141: 1167-76.

Rabilloud T, Blisnick T, Heller M, Luche S, Aebersold R, Lunardi J, et al. Analysis of membrane proteins by two-dimensional electrophoresis: comparison of the proteins extracted from normal or Plasmodium falciparum-infected erythrocyte ghosts. Electrophoresis. 1999; 20(18): 3603-10

Rhiel M, Bittl V, Tribensky A, Charnaud SC, Strecker M, Muller S, et al. Trafficking of the exported P. falciparum chaperone PfHsp70x. Sci Rep. 2016; 6: 36174.

Shevchenko A, Wilm M, Vorm O, Mann M. Mass spectrometric sequencing of proteins silver-stained polyacrylamide gels. Anal Chem. 1996; 68(5): 850-8.

Thermo Scientific. Proteome discoverer: user guide. Version 1.4. 2012. Available from: https://tools.thermofisher.com/content/ sfs/manuals/Man-XCALI-97506-Proteome-Discoverer-14-UserManXCALI97506-A-EN.pdf.

Vincensini L, Richert S, Blisnick T, Van Dorsselaer A, Leize-Wagner E, Rabilloud T, et al. Proteomic analysis identifies novel proteins of the Maurer's clefts, a secretory compartment delivering Plasmodium falciparum proteins to the surface of its host cell. Mol Cell Proteomics. 2005; 4(4): 582-93.

WHO - World Health Organization. World Malaria Report 2015. 2015. Available from: http://www.who.int/malaria/publications/worldmalaria-report-2015/report/en/.

Winter G, Kawai S, Haeggstrom M, Kaneko O, von Euler A, Kawazu $\mathrm{S}$, et al. SURFIN is a polymorphic antigen expressed on Plasmodium falciparum merozoites and infected erythrocytes. J Exp Med. 2005; 201(11): 1853-63. 